\title{
Arterial stiffness in symptomatic smokers with normal lung function
}

\author{
Nicola Scichilone ${ }^{1,2}$, Antonino Tuttolomondo ${ }^{1,2}$, Carlo Maida ${ }^{1}$, Alida Benfante ${ }^{1}$, \\ Jessica Peluso ${ }^{1}$, Anna Cirrincione ${ }^{1}$, Valerio Vassallo ${ }^{1}$ and Antonio Pinto ${ }^{1}$
}

Affiliations: 'Dept of Biomedicine and Internal and Specialistic Medicine, DIBIMIS, University of Palermo, Palermo, Italy. ${ }^{2}$ Both authors contributed equally.

Correspondence: Nicola Scichilone, DIBIMIS, University of Palermo, Via Trabucco 180, 90146 Palermo, Italy. E-mail: nicola.scichilonedunipa.it

ABSTRACT Chronic obstructive pulmonary disease (COPD) is associated with cardiovascular morbidity and mortality. Arterial stiffness and endothelial dysfunction index are validated surrogate cardiovascular markers and are increased in subjects with COPD.

We tested whether increased arterial stiffness and endothelial dysfunction occur in symptomatic smokers with no evidence of bronchial obstruction. Clinical and lung functional assessments were conducted in smoker subjects with chronic respiratory symptoms and in COPD patients. Pulse wave velocity (PWV), aortic augmentation index (AIx) and reactive hyperaemia index (RHI) were measured to estimate the cardiovascular risk.

48 smokers (male $\mathrm{n}=37$, female $\mathrm{n}=11$; mean age $70 \pm 8.4$ years) were studied. Smokers with respiratory symptoms without bronchial obstruction $(n=13)$ did not differ from COPD patients $(n=35)$ in terms of mean \pm SD PWV $\left(12 \pm 3.2 \mathrm{~m} \cdot \mathrm{s}^{-1}\right.$ versus $\left.14 \pm 3.8 \mathrm{~m} \cdot \mathrm{s}^{-1} ; \mathrm{p}=0.10\right)$, aortic AIx $(25.2 \pm 11.8 \%$ versus $24.8 \pm 9.7 \%$, $\mathrm{p}=0.89)$ and RHI $(1.66 \pm 1.49$ versus $1.62 \pm 1.43 ; \mathrm{p}=0.79)$.

The current investigation shows that the cardiovascular risk occurs in smokers with respiratory symptoms in the absence of bronchial obstruction. These findings need to be confirmed in larger populations in order to inform the design of intensive preventive programmes.

@ERSpublications

Increased cardiovascular risk occurs in smokers with chronic respiratory symptoms and no airway obstruction http://ow.ly/7mes30cCxW5

Cite this article as: Scichilone N, Tuttolomondo A, Maida C, et al. Arterial stiffness in symptomatic smokers with normal lung function. ERJ Open Res 2017; 3: 00037-2017 [https://doi.org/10.1183/ 23120541.00037-2017].

Received: March 222017 | Accepted after revision: June 022017

Conflict of interest: None declared

Copyright $\odot$ ERS 2017. This article is open access and distributed under the terms of the Creative Commons Attribution Non-Commercial Licence 4.0. 


\section{Introduction}

Chronic obstructive pulmonary disease (COPD) is among the most common diseases worldwide, and is predicted to become the third most common cause of death by the year 2020 [1]. The disease is characterised by a persistent and largely irreversible airflow limitation, and is associated with a massive local as well as systemic inflammatory response to inhalant pollutants, such as cigarette smoke [2]. Importantly, COPD is characterised by systemic complications that contribute to its morbidity and mortality; indeed, cardiovascular disorders are twice as common in individuals suffering from COPD than in subjects without COPD [3-5], and cardiovascular diseases are among the major causes of hospitalisation and mortality in COPD patients [4]. The mechanisms underlying this relationship are still largely unknown, and seem to be independent of smoke exposure and other confounding factors, such as age or degree of airway obstruction [6,7]. One proposed hypothesis lies in the loss of elasticity of the vascular structures [8], which eventually results in impaired shear stress and increased risk of atherosclerosis [9].

Experimental studies have reported that molecular and cellular pathways of inflammation can promote atherosclerosis. Risk factors for atherosclerosis can alter arterial biology, and produce a systemic milieu that favours atherothrombotic events [10]. For example, vascular cell adhesion molecule-1, expressed by cytokine-stimulated endothelial cells, binds inflammatory cells, such as monocytes and T-lymphocytes, that accumulate in the early atherosclerotic plaque. Furthermore, macrophages in atherosclerotic plaque progression express scavenger receptors necessary for the uptake of modified lipoproteins and hence foam-cell formation. The discovery that vascular wall cells themselves can produce cytokines, protein mediators of inflammation and immunity, provides an important insight into the initiation of atherosclerosis [11]. Inflammation regulates aspects of plaque biology that trigger the thrombotic complications of atherosclerosis. Arterial stiffness can be considered as a factor of progression of atherosclerosis and therefore an early marker of cardiovascular disease. Arterial stiffness has been demonstrated to be increased in COPD subjects $[6,7,12]$, suggesting a link between vascular and pulmonary diseases that may explain a proportion of the excess cardiovascular mortality in individuals affected by COPD. Arterial stiffness can be assessed noninvasively by estimation of aortic (carotid-femoral) pulse wave velocity (PWV), which is an established and robust marker of cardiovascular risk $[13,14]$ and has been associated with increased prevalence of COPD [15], and reduced lung function [7]. Endothelial dysfunction in peripheral arteries has been evaluated using forearm flow-mediated vasodilation (FMD) [16]. However, this technique offers findings that can vary widely due to technical problems, hence it is not a fully standardised method [17]. Kuvin et al. [18] described a new method to evaluate endothelial dysfunction: reactive hyperaemia peripheral arterial tonometry (RH-PAT), which measures hyperaemic response in a noninvasive way. In the current study, we explored whether cardiovascular risk, as expressed by increased arterial stiffness and endothelial dysfunction, occurs in the early stages of the disease, that is, in symptomatic smokers without signs of bronchial obstruction.

\section{Methods}

\section{Subjects}

Consecutive subjects referred to the outpatient respiratory clinics of the University of Palermo (Palermo, Italy) for chronic respiratory complaints were recruited if clinically stable. All enrolled patients had a documented history of cigarette smoke exposure (history of $\geqslant 10$ pack-years of smoking) and chronic respiratory complaints suggestive of COPD, such as chronic cough, exertional dyspnoea and chronic mucus production. Subjects were excluded if they were receiving long-term oxygen therapy, or had known cardiovascular, metabolic or systemic inflammatory diseases or malignancy, since they could interfere with the outcomes of the current study. Subjects were admitted to the study if their comorbid conditions were judged by the investigators not to influence the cardiovascular risk. None of the subjects were taking systemic anti-inflammatory drugs, such as oral corticosteroids.

The study consisted of two phases. In the first phase, clinical and lung functional assessments were performed in order to ascertain the diagnosis of COPD, according to international recommendations [2]. In the second phase, cardiovascular risk was evaluated. The study was conducted at the Department of Biomedicine and Internal and Specialistic Medicine at the University of Palermo. The study was approved by the local ethics committee, and written informed consent was obtained from each subject.

\section{Clinical and lung function assessments}

The clinical evaluation included the recording of respiratory symptoms and physical examination. Chronic respiratory symptoms suggestive of COPD, such as chronic cough, dyspnoea and chronic mucus production were recorded in all patients by means of specific questionnaires. In the present study, the modified Medical Research Council score was used to evaluate the degree of perception of dyspnoea. To 
comply with the scope of the study, we excluded subjects who reported documented features of cardiovascular and/or metabolic diseases.

Spirometry was performed in all subjects following the American Thoracic Society/European Respiratory Society task force guidelines [19]. Forced expiratory volume in $1 \mathrm{~s}$ (FEV1) and forced vital capacity (FVC), as well as forced expiratory flows at $25 \%, 50 \%$ and $75 \%$ of FVC were recorded for analysis. Bronchial obstruction was defined as a FEV1/FVC ratio below the lower limit of normal. All spirometric measurements were obtained from a computerised water-sealed spirometer (Biomedin, Padua, Italy), which allowed compliance with criteria for acceptability and reproducibility online. This phase took place in the morning. Subjects were then referred to the Department of Internal Medicine at the University of Palermo to undergo the second phase of the study, which was conducted within the same week.

\section{Cardiovascular risk assessment}

Arterial stiffness was investigated using noninvasive tests in a temperature-controlled room, with the subject resting in supine position. The carotid-femoral PWV was measured by specifically trained staff using applanation tonometry (SphygmoCor; AtCor Medical, Sydney, Australia) at the carotid and radial arteries to record the pressure waveforms peripherally. The correlation between PWV and arterial distensibility is based on the calculation of the speed of the propagation of transverse elastic waves. By applying the integral software, the aortic augmentation index (AIx; the quotient of amplification pressure on pulse pressure expressed as a percentage) was calculated. Quality control checks were made for all measurements to discharge measurements in which the onset of the waveform was not clearly identified.

The endothelial dysfunction was evaluated using the EndoPAT 2000 (Itamar Medical, Columbia, SC, USA), which measures the change in brachial artery diameter in response to reactive hyperaemia after an overnight fast. The hyperaemia was induced by deflating a cuff placed around the forearm that had been inflated for $5 \mathrm{~min}$; the reactive hyperaemia response was calculated automatically by the instrument, thus providing the reactive hyperaemia index (RHI), which was recorded for the analysis.

\section{Statistical analysis}

The statistical package used was StatView 5.0.1 (Abacus Concept, Berkeley, CA, USA). Data are presented as mean $\pm \mathrm{SD}$ or median (range), depending on whether they were normally distributed. Differences between groups were tested using the t-test or Mann-Whitney U-test, as appropriate. Differences at p-values $<0.05$ were considered statistically significant.

\section{Results}

The study included 48 subjects (male $n=37$, female $n=11$; mean age $70 \pm 8.4$ years); mean systolic blood pressure was $127 \pm 13 \mathrm{mmHg}$ and diastolic blood pressure was $81 \pm 8 \mathrm{mmHg}$. 35 individuals were classified as COPD patients (obstructed group), whereas the remaining 13 subjects were experiencing respiratory symptoms, such as exertional dyspnoea and chronic cough, without any spirometric feature of airway obstruction (nonobstructed group). The two groups had comparable ages (71.3 \pm 6.7 versus $66 \pm 11.3$ years), smoke exposure $(57 \pm 40$ versus $75 \pm 66$ pack-years), number of comorbidities $(4.1 \pm 2.1$ versus $4.3 \pm 1.8)$ and body mass index $\left(28.2 \pm 5\right.$ versus $\left.28.3 \pm 5.3 \mathrm{~kg} \cdot \mathrm{m}^{-2}\right)$, with $\mathrm{p}>0.5$ in all comparisons. The most frequent comorbidities were depression, osteoporosis, glaucoma and/or cataracts and muscle wasting. By design, lung functional evaluation showed significant differences between the two study groups (table 1).

When we assessed the cardiovascular risk, no differences between obstructed and nonobstructed subjects were found. In particular, PWV was $14 \pm 3.8 \mathrm{~m} \cdot \mathrm{s}^{-1}$ and $12 \pm 3.2 \mathrm{~m} \cdot \mathrm{s}^{-1}$, respectively; $\mathrm{p}=0.10$ ) (figure 1a).

\section{TABLE 1 Lung functional characteristics of nonobstructed and obstructed subjects}

\begin{tabular}{lcc} 
& Nonobstructed & Obstructed \\
\hline FEV 1 \% pred & $103 \pm 15$ & $64 \pm 17$ \\
FVC \% pred & $106 \pm 12$ & $83 \pm 17$ \\
FEV 1 FVC & $0.77 \pm 0.4$ & $0.60 \pm 0.8$ \\
FEF25\% \% pred & $81 \pm 23$ & $32 \pm 18$ \\
FEF50\% \% pred & $100 \pm 41$ & $29 \pm 11$ \\
FEF75\% \% pred & $84 \pm 36$ & $41 \pm 18$ \\
FEF25-75\% \% pred & $61 \pm 5$ & $27 \pm 10$
\end{tabular}

Data are presented as mean \pm SD. FEV1: forced expiratory volume in $1 \mathrm{~s}$; FVC: forced vital capacity; FEFx\%: forced expiratory flow at $25 \%, 50 \%$ and $75 \%$ of FVC. $p<0.0001$ for all comparisons. 

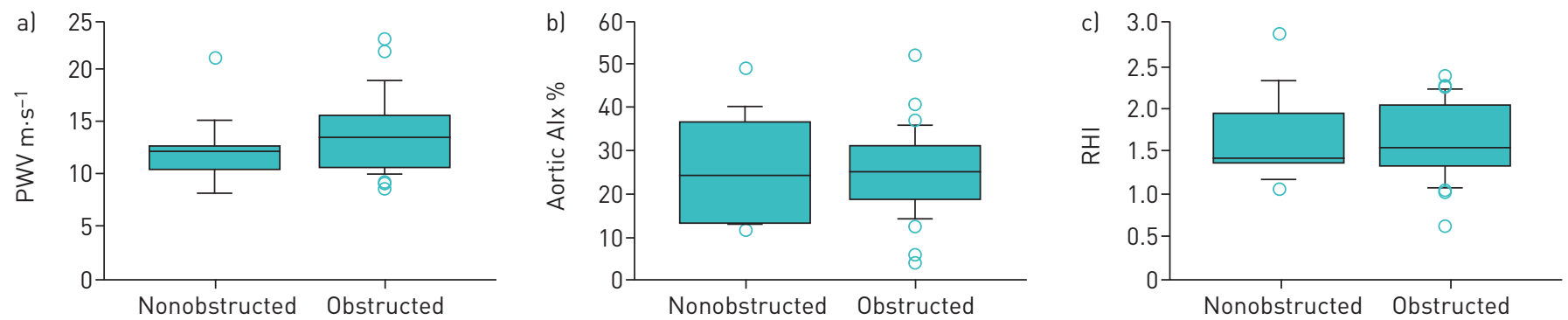

FIGURE 1 Differences between nonobstructed and obstructed subjects in terms of a) pulse wave velocity (PWV); b) aortic augmentation index (Alx); c) reactive hyperaemia index (RHI).

Similarly, the aortic AIx was $24.8 \pm 9.7 \%$ and $25.2 \pm 11.8 \%$, respectively ( $\mathrm{p}=0.89$ ), and RHI was $1.62 \pm 1.4$ versus $1.66 \pm 1.5 \quad(p=0.79)$ (figure $1 \mathrm{~b}$ and $\mathrm{c}$ ). There was no association between age or pack-years of smoking with any marker of cardiovascular risk in either group or the entire study sample $(\mathrm{p}>0.05$ in all analyses).

\section{Discussion}

The findings of the current study confirm and extend the knowledge on the relationship between markers of cardiovascular risk and COPD, by demonstrating that increased arterial stiffness and endothelial dysfunction occur in symptomatic, nonobstructed smokers. The demonstration of higher levels of arterial stiffness and endothelial dysfunction in symptomatic subjects who do not show any sign of airway obstruction, to the same extent as obstructed patients, is novel and deserves some attention. Indeed, it identifies an excess risk for cardiovascular events, even in subjects who have not developed lung function impairment, implying in addition that arterial stiffness is not dependent on the occurrence (and magnitude) of airflow limitation. The current observations carry pathophysiological consequences, in that they allow speculation on the link between pulmonary and cardiovascular diseases. We favour the hypothesis that long-lasting exposure to smoke is the driving factor that initiates the inflammatory process both in the lungs and in the arteries, which in turn promotes the excess cardiovascular risk in chronic respiratory patients.

Patients with COPD have peripheral lung inflammation, and they show increased circulating cytokines, including interleukin (IL)-6, IL-18 and TNF- $\alpha$, as well as acute-phase proteins, such as C-reactive protein [20]. This low-grade chronic systemic inflammation may explain the increased propensity to cardiovascular, metabolic, bone and neurological diseases. Systemic inflammation in COPD is known to promote atherosclerosis [21]. In this context, arterial stiffness is a useful noninvasive measure of vascular function, and is associated with atheromatous changes in the vascular bed [22]. The viscoelastic properties of the arteries allow propagation of the pulse wave along vessel walls generated from left ventricular ejection. The speed of this wave is directly linked to arterial stiffness. Early studies [23-25] demonstrated that arterial stiffness is an independent predictor of cardiovascular accidents in other chronic pathological conditions, such as diabetes, renal failure and systemic hypertension. In individuals suffering from COPD, in contrast to healthy age-matched controls, arterial stiffness has been shown to be increased [12], and to correlate with the degree of airflow limitation [7]. In a cohort of 157 patients with COPD, MCALLISTER et al. [6] found that the extent of arterial stiffness was associated with the severity of emphysema, suggesting similar pathophysiological processes in both the lungs and the vascular tissue. Furthermore, studies using the EndoPAT have shown that the RHI score reflects bioavailability of nitric oxide [26].

Endothelial dysfunction in peripheral arteries is assessed by forearm flow-mediated vasodilation [16]. However, the results of forearm flow-mediated vasodilation can vary due to technical problems encountered during measurement, and thus it is not standardised among institutions [16]. KUVIN et al. [18] described a new method to evaluate endothelial dysfunction (RH-PAT). It is a noninvasive, automatic and quantitative clinical test for digital measurement of hyperaemic response. Using this test, the Framingham Heart Study reported RHI as inversely correlated with various cardiovascular risk factors [18]. Impaired RHI is a noninvasive measure of peripheral vascular dysfunction that can identify individuals with acetylcholine-induced vascular dysfunction. Endothelial dysfunction is one of the earliest events in the pathophysiological process leading to atherosclerotic disorders [27], and contributes to the progression of disease by means of inflammation and thrombosis $[28,29]$. The RHI correlates with the measurement of endothelial vasodilator function in the coronary arteries [28] and with brachial FMD [19]. Patients with a greater degree of cardiovascular disease exhibit a lower score, and values are lower in other conditions associated with impaired endothelial function and risk of cardiovascular disease [30, 31] 
Therefore, in our study arterial stiffness and RHI represent two validated surrogate cardiovascular outcomes which depict the cardiovascular risk profile of subjects with pulmonary disease; our findings corroborate the notion that arterial stiffening and endothelial dysfunction develop precociously in COPD subjects independently from clinical and functional severity of lung disease.

The mechanisms responsible for higher arterial stiffness in patients with COPD are largely unknown. The most plausible hypothesis is that, similar to that proposed for other chronic inflammatory conditions [23, 25], arterial stiffness is related to the state of chronic systemic inflammation that follows local (lung) inflammation as a consequence of "spill over" of the inflammatory process into the systemic circulation [32]. Indeed, fibrinogen levels $[33,34]$ and C-reactive protein $[35,36]$ are higher in COPD patients than in control subjects. However, the possibility that systemic inflammation due to causative factors such as smoking exposure eventually affect the airways, thus leading to inflammatory changes that predispose to COPD, cannot be ruled out. An interesting hypothesis is that both impaired lung function and increased arterial stiffness in COPD are due to the increased susceptibility to degradation of connective tissue, as an expression of premature ageing triggered by smoking exposure. Elastolytic activity by means of metalloproteinases is enhanced in emphysema and atherosclerosis: on one hand, elastin degradation leads to loss of alveolar attachments and emphysema [9]; on the other hand, elastin degradation is associated with increased collagen and thicker arteries [37].

In the interpretation of the results of the current study, some limitations should be taken into account. First, we did not have access to laboratory tests to rule out the occurrence of metabolic syndrome or other inflammatory diseases. However, we were careful to exclude patients with clear signs and/or symptoms of systemic diseases (comorbidities) that could interfere with the outcomes of the study. Obviously, the possibility of asymptomatic diseases or mild forms of comorbid conditions that may contribute to arterial stiffness cannot be completely excluded. Second, our sample was relatively small and we cannot exclude that the lack of significant differences between the two groups is to be attributed to low statistical power. Therefore, the present findings need confirmation with investigations conducted in larger populations and with a longitudinal design; if they hold true, intensive preventive programmes specifically designed for the early stages of COPD should be strongly advocated. In these patients, smoking cessation, promotion of physical activity and body weight reduction and pharmacological treatment of cardiovascular risk factor such as hypertension, dyslipidaemia and high glucose blood levels are useful strategies to reduce the cardiovascular risk. In conclusion, these observations suggest that systemic complications of COPD occur at the early stages of the disease, and increase with progression of lung function decline, perhaps representing a premature ageing effect.

\section{References}

1 Murray CJ, Lopez AD. Global mortality, disability, and the contribution of risk factors: Global Burden of Disease Study. Lancet 1997; 349: 1436-1442.

2 GOLD Executive Committee. Global Strategy for the Diagnosis, Management and Prevention of Chronic Obstructive Pulmonary Disease 2017 Report. Date last accessed: February 14, 2017. Date last updated: January 20, 2017.

3 Sin DD, Wu L, Man SF. The relationship between reduced lung function and cardiovascular mortality: a population-based study and a systematic review of the literature. Chest 2005; 127: 1952-1959.

4 Huiart L, Ernst P, Suissa S. Cardiovascular morbidity and mortality in COPD. Chest 2005; 128: $2640-2646$.

5 Rabe KF, Cooper CB. Global Initiative on Obstructive Lung Disease revised: what constitutes a guideline? Am J Respir Crit Care Med 2013; 187: 1035-1036.

6 McAllister DA, Maclay JD, Mills NL, et al. Arterial stiffness is independently associated with emphysema severity in patients with chronic obstructive pulmonary disease. Am J Respir Crit Care Med 2007; 176: 1208-1214.

7 Costanzo L, Pedone C, Battistoni F, et al. Relationship between FEV1 and arterial stiffness in elderly people with chronic obstructive pulmonary disease. Aging Clin Exp Res 2017; 29: 157-164.

8 Maclay JD, MacNee W. Cardiovascular disease in COPD: mechanisms. Chest 2013; 143: 798-807.

9 Zieman SJ, Melenovsky V, Kass DA. Mechanisms, pathophysiology, and therapy of arterial stiffness. Arterioscler Thromb Vasc Biol 2005; 25: 932-943.

10 Ross R, Glomset JA. The pathogenesis of atherosclerosis (first of two parts). N Engl J Med 1976; 295: 369-377.

11 Ross R, Glomset JA. The pathogenesis of atherosclerosis (second of two parts). N Engl J Med 1976; 295: $420-425$.

12 Sabit R, Bolton CE, Edwards PH, et al. Arterial stiffness and osteoporosis in chronic obstructive pulmonary disease. Am J Respir Crit Care Med 2007; 175: 1259-1265.

13 Meaume S, Benetos A, Henry OF, et al. Aortic pulse wave velocity predicts cardiovascular mortality in subjects $>70$ years of age. Arterioscler Thromb Vasc Biol 2001; 21: 2046-2050.

14 Laurent S, Cockcroft J, Van Bortel L, et al. Expert consensus document on arterial stiffness: methodological issues and clinical applications. Eur Heart J 2006; 27: 2588-2605.

15 Mills NL, Miller JJ, Anand A, et al. Increased arterial stiffness in patients with chronic obstructive pulmonary disease: a mechanism for increased cardiovascular risk. Thorax 2008; 63: 306-311.

16 Widlansky ME, Gokce N, Keaney JF Jr, et al. The clinical implications of endothelial dysfunction. J Am Coll Cardiol 2003; 42: 1149-1160.

17 Hijmering ML, Stroes ES, Pasterkamp G, et al. Variability of flow mediated dilation: consequences for clinical application. Atherosclerosis 2001; 157: 369-373. 
Kuvin JT, Patel AR, Sliney KA, et al. Assessment of peripheral vascular endothelial function with finger arterial pulse wave amplitude. Am Heart J 2003; 146: 168-174.

19 Pellegrino R, Viegi G, Brusasco V, et al. Interpretative strategies for lung function tests. Eur Respir J 2005; 26: 948-968.

20 Boschetto P, Beghé B, Fabbri LM, et al. Link between chronic obstructive pulmonary disease and coronary artery disease: implication for clinical practice. Respirology 2012; 17: 422-431.

21 Sin DD, Man SFP. Why are patients with chronic obstructive pulmonary disease at increased risk of cardiovascular diseases? The potential role of systemic inflammation in chronic obstructive pulmonary disease. Circulation 2003; 107: 1514-1519.

22 Van Popele NM, Mattace-Raso FU, Vliegenthart R, et al. Aortic stiffness is associated with atherosclerosis of the coronary arteries in older adults: the Rotterdam Study. J Hypertens 2006; 24: 2371-2376.

23 Cruickshank K, Riste L, Anderson SG, et al. Aortic pulse-wave velocity and its relationship to mortality in diabetes and glucose intolerance: an integrated index of vascular function? Circulation 2002; 106: 2085-2090.

24 Blacher J, Guerin AP, Pannier B, et al. Impact of aortic stiffness on survival in end-stage renal disease. Circulation 1999; 99: 2434-2439.

25 Boutouyrie P, Tropeano AI, Asmar R, et al. Aortic stiffness is an independent predictor of primary coronary events in hypertensive patients: a longitudinal study. Hypertension 2002; 39: 10-15.

26 Nohria A, Gerhard-Herman M, Creager MA, et al. Role of nitric oxide in the regulation of digital pulse volume amplitude in humans. J Appl Physiol 2006; 101: 545-548.

27 Davies PF. Endothelial mechanisms of flow-mediated athero-protection and susceptibility. Circ Res 2007; 101: 10-12.

28 Libby P, Ridker PM, Hansson GK. Inflammation in atherosclerosis: from pathophysiology to practice. $J$ Am Coll Cardiol 2009; 54: 2129-2138.

29 Lamon BD, Hajjar DP. Inflammation at the molecular interface of atherogenesis: an anthropological journey. Am J Pathol 2008; 173: 1253-1264.

30 Hirata Y, Nagata D, Suzuki E, et al. Diagnosis and treatment of endothelial dysfunction in cardiovascular disease. Int Heart J 2010; 51: 1-6.

31 Hamburg NM, Keyes MJ, Larson MG, et al. Cross-sectional relations of digital vascular function to cardiovascular risk factors in the Framingham Heart Study. Circulation 2008; 117: 2467-2474.

32 Gan WQ, Man SF, Senthilselvan A, et al. Association between chronic obstructive pulmonary disease and systemic inflammation: a systematic review and a meta-analysis. Thorax 2004; 59: 574-580.

33 Dahl M, Tybjaerg-Hansen A, Vestbo J, et al. Elevated plasma fibrinogen associated with reduced pulmonary function and increased risk of chronic obstructive pulmonary disease. Am J Respir Crit Care Med 2001; 164 1008-1011.

34 Engström G, Lind P, Hedblad B, et al. Lung function and cardiovascular risk: relationship with inflammation-sensitive plasma proteins. Circulation 2002; 106: 2555-2560.

35 Mendall MA, Strachan DP, Butland BK, et al. C-reactive protein: relation to total mortality, cardiovascular mortality and cardiovascular risk factors in men. Eur Heart J 2000; 21: 1584-1590.

36 Pinto-Plata VM, Müllerova H, Toso JF, et al. C-reactive protein in patients with COPD, control smokers and non-smokers. Thorax 2006; 61: 23-28.

37 Brooke BS, Bayes-Genis A, Li DY. New insights into elastin and vascular disease. Trends Cardiovasc Med 2003; 13: $176-181$. 